\title{
Parecoxib Added to Ropivacaine Prolongs Duration of Axillary Brachial Plexus Blockade and Relieves Postoperative Pain
}

\author{
Xiaoming Liu PhD, Xuan Zhao MD, \\ Jian Lou MD, Yingwei Wang PhD, Xiaofang Shen MD
}

Received: 29 February 2012 / Accepted: 26 October 2012/Published online: 21 November 2012

(C) The Association of Bone and Joint Surgeons (B) 2012

\begin{abstract}
Background Cyclooxygenase (COX)-2 antagonist is widely used for intravenous postoperative pain relief. Recent studies reported COX-2 in the spinal dorsal horn could modulate spinal nociceptive processes. Epidural parecoxib in rats showed no neurotoxicity. These findings suggested applying a COX-2 antagonist directly to the central or peripheral nerve might provide better analgesia. Questions/purposes We therefore determined: (1) whether the addition of parecoxib to ropivacaine injected locally on the nerve block affected the sensory and motor block times of the brachial plexus nerve block; and (2) whether parecoxib injected locally on the nerve or intravenously had a similar analgesic adjuvant effect.
\end{abstract}

Each author certifies that he or she, or a member of his or her immediate family, has no funding or commercial associations (eg, consultancies, stock ownership, equity interest, patent/licensing arrangements, etc) that might pose a conflict of interest in connection with the submitted article.

All ICMJE Conflict of Interest Forms for authors and Clinical Orthopaedics and Related Research editors and board members are on file with the publication and can be viewed on request.

Clinical Orthopaedics and Related Research neither advocates nor endorses the use of any treatment, drug, or device. Readers are encouraged to always seek additional information, including FDA-approval status, of any drug or device prior to clinical use. Each author certifies that his or her institution approved the human protocol for this investigation, that all investigations were conducted in conformity with ethical principles of research, and that informed consent for participation in the study was obtained.

X. Liu, X. Zhao (凹), J. Lou, Y. Wang, X. Shen Department of Anesthesiology, Xinhua Hospital, Shanghai Jiaotong University School of Medicine, 200092 Shanghai, China

e-mail: zhaoxuan0323@hotmail.com
Methods We conducted a randomized controlled trial from January 2009 to November 2010 with 150 patients scheduled for elective forearm surgery, using a multiplenerve stimulation technique. Patients were randomly allocated into one of three groups: Group A $(n=50)$ received ropivacaine $0.25 \%$ alone on the brachial plexus nerve; Group B $(n=50)$ received ropivacaine together with $20 \mathrm{mg}$ parecoxib locally on the nerve block; and Group C $(\mathrm{n}=50)$ received $20 \mathrm{mg}$ parecoxib intravenously. We recorded the duration of the sensory and motor blocks, and the most severe pain score during a 24-hour postoperative period.

Results Parecoxib added locally on the nerve block prolonged the motor and sensory block times compared with Group A. However, parecoxib injected intravenously had no such effect. Pain intensity scores in Group B were lower than those in Groups A and C.

Conclusions Parecoxib added to ropivacaine locally on the nerve block prolonged the duration of the axillary brachial plexus blockade and relieved postoperative pain for patients having forearm orthopaedic surgery.

Level of Evidence Level I, therapeutic study. See Guidelines for Authors for a complete description of levels of evidence.

\section{Introduction}

An axillary brachial plexus nerve block is used frequently for forearm surgery [3]. A peripheral nerve block has less of an effect on the cardiovascular and pulmonary systems [3] and provides faster postoperative rehabilitation compared with general anesthesia [11], therefore it is preferable for some patients. The multiple-nerve stimulation technique, in which the four main nerves of the plexus are localized at the axilla 
by a nerve stimulator and separately injected, reportedly provides a short onset time [24, 25]. Zhao et al. showed that a complete block could be obtained in $94.4 \%$ of patients receiving a low concentration of levobupivacaine $(36 \mathrm{~mL}$, $0.1 \%$ ) using this technique [30]. These findings confirmed that a multiple-nerve stimulation technique is reasonable for orthopaedic surgery of the forearm.

Parecoxib, a kind of cyclooxygenase (COX)-2 antagonist, rapidly hydrolyzed in vivo to its active form, valdecoxib [10]. Several clinical trials have indicated a single dose of parecoxib at $40 \mathrm{mg}$ had a similar analgesic effect as ketorolac in treating postoperative pain resulting from oral surgery [6], orthopaedic surgery [23], and abdominal hysterectomy [20]. Other studies [12, 21, 28] have shown no major effects on platelet function or upper gastrointestinal mucosa. Intravenous use of a COX-2 antagonist may inhibit the systemic inflammatory response and prostaglandin synthesis, relieve inflamed peripheral tissues, and consequently provide pain relief [26]. Two studies showed that COX-2 in the spinal dorsal horn could modulate spinal nociceptive processes and is associated with antihyperalgesia in the central nervous system [16, 17]. Therefore, we postulated that applying a COX-2 antagonist directly to the peripheral nerve might reduce the COX-2 activation and down-regulate pain intensity.

We therefore determined: (1) whether the addition of parecoxib to ropivacaine injected locally on the nerve block affected the sensory and motor block times of the brachial plexus nerve block; and (2) whether parecoxib injected locally on the brachial plexus nerve or injected intravenously had a similar analgesic adjuvant effect or which one was better.

\section{Patients and Methods}

We enrolled all 150 patients scheduled for elective surgery of the hand, wrist, or forearm from January 2009 to November 2010. The inclusion criteria were: American Society of Anesthesiologists (ASA) Class I to II [14], between 18 and 65 years old, weight between 45 and $90 \mathrm{~kg}$, height greater than $150 \mathrm{~cm}$, and first surgery of the forearm. We excluded 100 patients for whom an axillary brachial plexus block or the study medications were contraindicated or who had a history of major neurologic or psychiatric disorder, diabetes, stroke, neuromuscular, cardiovascular, pulmonary, renal, or hepatic disease; alcohol or drug abuse, and pregnant or lactating women. Patients not having their first forearm surgery, such as removal of internal fixation, were excluded. These 100 exclusions left 150 patients for study (50 patients per group) (Fig. 1). Patients enrolled were all inpatients who would remain in the hospital for at least 5 days after surgery. All subjects reported no preexisting pain and did not take any analgesic medications before surgery. None of the 150 patients had a clinical psychologic disorder or pain-relevant comorbidities. The time from diagnosis to treatment of all patients was within 3 months. The operative procedures included débridement and suturing of the forearm or hand, carpal tunnel release, cubital tunnel release, open reduction and internal fixation (ORIF) of an ulna fracture, radius fracture, or fracture of both bones, distal radius fracture, olecranon fracture, radial head fracture, and ORIF of a metacarpal fracture (Table 1).

By using a computer-generated sequence of random numbers, patients were assigned to one of the three groups. All blocks were performed using a multiple-nerve stimulation technique in which the four main nerves of the plexus are localized at the axilla by a nerve stimulator and separately injected. Group A $(n=50)$ received a total of $40 \mathrm{~mL}$ ropivacaine $0.25 \%$, and $4 \mathrm{~mL}$ normal saline (NS) on the nerve block $(10 \mathrm{~mL}$ ropivacaine and $1 \mathrm{~mL}$ NS for each nerve) and $5 \mathrm{~mL}$ NS intravenously. Group B $(\mathrm{n}=50)$ received a total of $40 \mathrm{~mL}$ ropivacaine and $20 \mathrm{mg}$ parecoxib (diluted in $4 \mathrm{~mL} \mathrm{NS}$ ) and $5 \mathrm{~mL} \mathrm{NS}$ intravenously. The volume for each nerve block was $10 \mathrm{~mL}$ ropivacaine and $5 \mathrm{mg}$ parecoxib. Group $\mathrm{C}(\mathrm{n}=50)$ received a total of $40 \mathrm{~mL}$ ropivacaine and $4 \mathrm{~mL}$ NS together with $20 \mathrm{mg}$ parecoxib (diluted in $5 \mathrm{~mL} \mathrm{NS}$ ) intravenously. The volume for each nerve block was $10 \mathrm{~mL}$ ropivacaine and $1 \mathrm{~mL}$ NS. No adrenaline was added to the solutions. One author (YW) was in charge of the random allocation. The patients, other authors, medical staff, and the statistician were unaware of treatment group assignments. The study protocol was approved by the Shanghai Jiaotong University Xinhua Hospital Ethics Committee (approved on December 25, 2008; XHEC-D-2008-035). Each patient read and signed a consent form before enrollment in the study.

The sample size was calculated according to results of a pilot study performed at our hospital. The control group and the local parecoxib group each had 10 patients. The VAS $(0 \mathrm{~mm}=$ no pain; $100 \mathrm{~mm}=$ worst imaginable pain) scores and sensory block time were recorded for all patients. A sample size of at least 37 patients in each group would be necessary to provide $80 \%$ power and a $5 \% \alpha$ error to detect a difference of 74 minutes on sensory block time and $25 \mathrm{~mm}$ on the VAS between groups (PASS 2008 software, NCSS LLC, Kaysville, UT, USA).

Patients were premedicated with $1 \mathrm{mg}$ midazolam intravenously in the operating room immediately before the surgery started. Standard monitors including noninvasive arterial blood pressure, electrocardiograph (Lead II), heart rate, and oxygen saturation were recorded. A nurse anesthetist (YQ) not involved in the performance of the brachial plexus block, patient care, and data collection, prepared all local anesthetic solutions and drugs. All nerve blocks were 


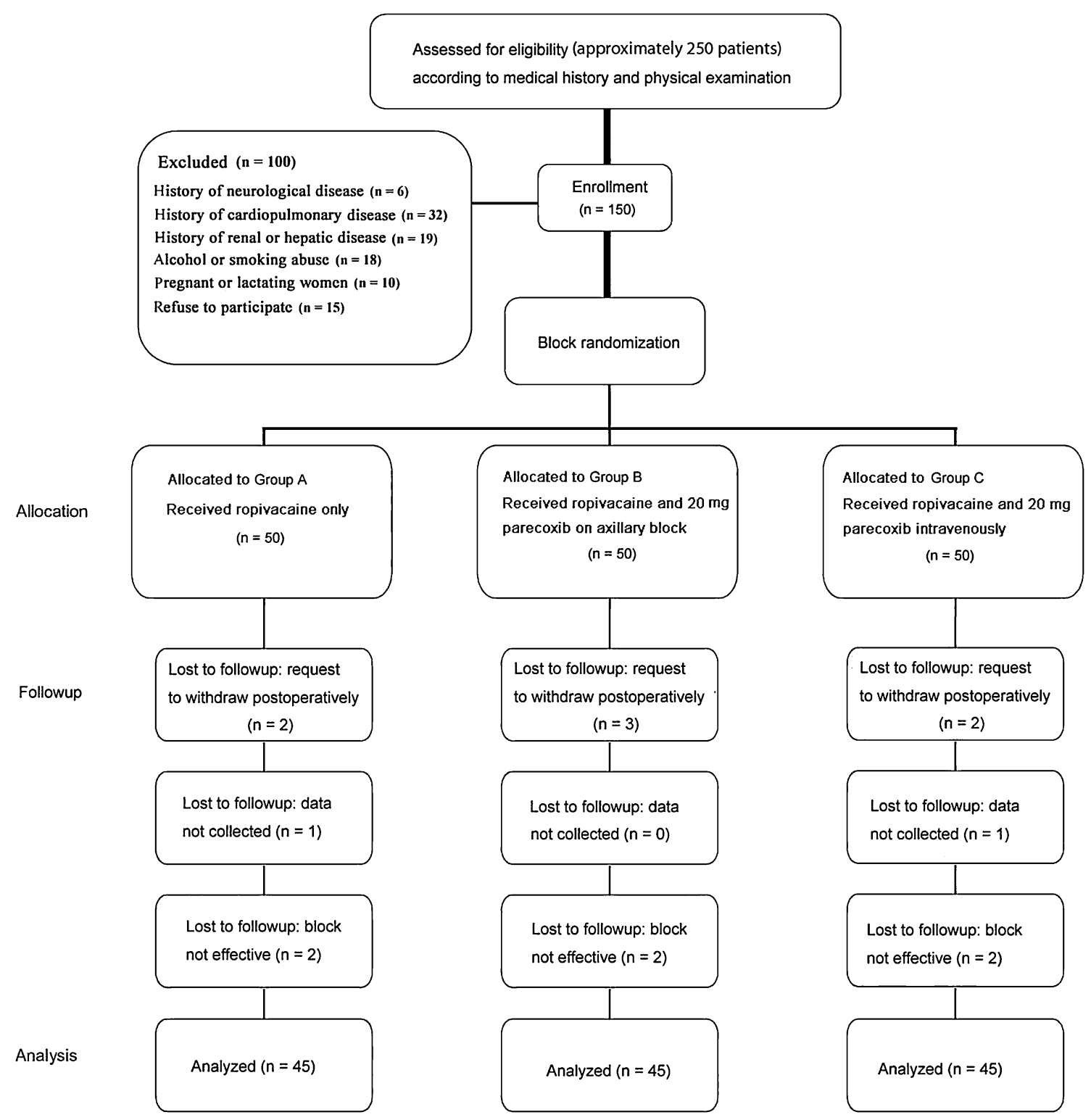

Fig. 1 The diagram shows the flow of patients treated with different interventions.

performed by the same experienced anesthesiologist (XZ) with the aid of a nerve stimulator (Stimuplex DIG ${ }^{\circledR}$; B. Braun, Melsungen, Germany). Patients were asked to abduct their arm to an angle of $90^{\circ}$. The arterial pulse was palpated at the level of the pectoralis major muscle crossing the axilla. A 22-gauge, 50-mm stimulating needle (Stimuplex ${ }^{\circledR}$; B. Braun) was inserted. The nerve stimulator initially was set at a current output of $1.0 \mathrm{~mA}$, frequency of $1 \mathrm{~Hz}$, and pulse duration of $0.1 \mathrm{~ms}$. Nerves were located according to specific twitches elicited by their stimulation (musculocutaneous nerve $=$ forearm flexion; median $=$ radial flexion of the wrist, second and third finger flexion, pronation; ulnar $=$ ulnar flexion of the wrist, fourth and fifth finger flexion, thumb adduction; radial $=$ wrist and/or finger extension with or without forearm extension). After obtaining a visible motor response at a current output of less than $0.5 \mathrm{~mA}$ and negative response lower than $0.2 \mathrm{~mA}$ and followed by a negative aspiration test, the predetermined ropivacaine and NS or parecoxib were injected. After injection, the arm was kept adducted with the hand resting on the chest. Sensory and motor block assessments were evaluated before surgery according to the literature $[9,13]$. One of us (XS) evaluated the sensory and motor blocks of the musculocutaneous, radial, ulnar, and median nerve immediately and at 5, 10, 20, and 30 minutes after completion of the injection. At every timepoint, the sensory and motor blocks were evaluated three times. Briefly, the sensory block was determined by the pinprick test. A 
Table 1. Detailed characterization of surgical procedures (absolute numbers)

\begin{tabular}{lccc}
\hline Surgical procedure & Group A & Group B & Group C \\
\hline $\begin{array}{l}\text { Débridement and suture of the hand } \\
\quad 5\end{array}$ & 5 & 3 \\
$\quad$ and/or forearm & 2 & 2 & 2 \\
Carpal tunnel release & 1 & 0 & 1 \\
Cubital tunnel release & 2 & 2 & 2 \\
ORIF of first metacarpal fracture & 1 & 1 & 1 \\
ORIF of second metacarpal fracture & 1 & 1 & 0 \\
ORIF of fifth metacarpal fracture & 1 & 7 & 7 \\
ORIF of ulna fracture & 6 & 7 & 7 \\
ORIF of radius fracture & 6 & 10 & 10 \\
ORIF of ulna and radius fracture & 10 & 4 & 4 \\
ORIF of distal radius fracture & 4 & 4 & 4 \\
ORIF of olecranon fracture & 4 & 4 & 4 \\
ORIF of radial head fracture & 3 & & \\
\hline
\end{tabular}

ORIF $=$ open reduction internal fixation.

pinprick sensation on the contralateral arm was scored as 100 points. Patients were requested to compare pinpricks (27-gauge needle) in the primary innervation areas of the respective nerves in the anesthetized arm with the contralateral arm. The scale ranged from 100 points (full sensation) to 0 points (no sensation). The sensory areas in the skin were the lateral side of the forearm (musculocutaneous nerve), thenar eminence (median nerve), hypothenar eminence (ulnar nerve), and radial dorsum of the hand (radial nerve). Primary block effectiveness was calculated as the percentage of patients in each group in which an effective block was obtained at 30 minutes. After 30 minutes of evaluation, if any of the major nerves involved in the planned surgical intervention had a sensibility greater than 20 points, they were regarded as having a failed block and were excluded from further investigation. Six patients had failed blocks (two in Group A, two in Group B, and two in Group C) in our study, and received supplementary blocks $(5 \mathrm{~mL}$ levobupivacaine $0.25 \%)$ and were excluded from the study. Motor block also was evaluated according to previous studies $[9,13]$. Measurements were performed using a modification of the Lovett rating scale from 6 (normal muscle force) to 0 points (complete paralysis) by extending the thumb at the interphalangeal joint (radial nerve), thumb adduction (ulnar nerve), thumb opposition (median nerve), and flexion of the elbow in supination and pronation of the forearm (musculocutaneous nerve). Motor block effectiveness was defined as a reduction of muscle force to 3 or less. In the current study, only one of us was in charge of sensory and motor assessments. Therefore we cannot provide the interobserver variability. However, the interobserver reliability of pinprick sensation was $81 \%$ [18] and $72 \%$ to $93 \%$ of the Lovett scale [4], according to previous studies.

Onset time and end time of anesthesia (nerve block), onset time and end time of the operation, and latency time (from the end of the primary block to time the patient was declared ready for surgery) were recorded by the anesthesiologist during surgery.

Two patients in Group A, three in Group B, and two in Group C with successful blocks requested to withdraw from evaluation after surgery and therefore were omitted from analysis. One patient in Group A and one in Group C who did not complete the data collection process after surgery also were omitted. This left sample sizes of 45 participants in each group.

At the end of surgery, the patients were informed that they should note the time, in their opinion, when the motor function or sensation of the arm was fully recovered from the block separately. Patients were instructed to call our service telephone and the same investigator (JL), would go to the ward and check the patient's sensory and motor function and record the time. The investigator (JL) would also go to the ward to check the patients every 2 hours after surgery no matter whether the patients called or not. At each visit, the pinprick sensation test versus contralateral arm and Lovett scale for motor test were assessed by this investigator and the operating surgeon. After the patient's sensation was fully recovered, the incision pain scores were recorded. The investigator would ask all patients to rate the incision pain intensity score on a 100-mm-long VAS $(0 \mathrm{~mm}=$ no pain; $100 \mathrm{~mm}=$ worst imaginable pain $)$. Postoperative parameters recorded by the investigator (JL) were time of complete sensory recovery and of complete motor recovery, and the intensity of the most severe pain (VAS score) within 24 hours after surgery.

Anesthesia-related complications and their grades also were recorded by one of us (JL) and the operating surgeon. Common neurovascular complications for axillary brachial plexus nerve block included systemic toxicity of local anesthetics, puncture of the axillary artery, hematoma formation, and sensory paresthesia during the procedure or dysesthesia after surgery [27]. The first three were attributed to unintentional intravascular injection of local anesthetic. Postoperative dysesthesia was recorded 24 hours after the operation and again 2 months later. The grades of complications were determined according to the classification of Dindo et al. [8].

Patient characteristics were compared by ANOVA and chi-square test for multiple groups. Analysis of patient characteristics showed no differences among groups (Table 2). Durations of motor and sensory blocks were compared for variability across groups using Levene's test. These data were performed for the normal distribution test by the Shapiro-Wilk test. Because distribution was 
Table 2. Patient characteristics (absolute numbers, percentage, or mean [SD], or median [minimum-maximum])

\begin{tabular}{llll}
\hline Characteristic & Group A & Group B & Group C \\
\hline Sex (M/F) & $26 / 19$ & $23 / 22$ & $24 / 21$ \\
Age (years) & $40(15)$ & $45(19)$ & $41(17)$ \\
$\begin{array}{l}\text { ASA physical status } \\
\quad(\mathrm{I} / \mathrm{II})\end{array}$ & $33 / 12$ & $34 / 11$ & $30 / 15$ \\
$\begin{array}{c}\text { Region of surgery } \\
\quad \text { forearm/hand) }\end{array}$ & $35 / 10$ & $37 / 8$ & $37 / 8$ \\
$\begin{array}{c}\text { Type of surgery } \\
\quad \text { (bone/soft tissue) }\end{array}$ & $37 / 8$ & $40 / 5$ & $39 / 6$ \\
$\begin{array}{c}\text { Duration of surgery } \\
\quad(\text { minutes) }\end{array}$ & $73(20-182)$ & $80(14-156)$ & $67(24-152)$ \\
$\begin{array}{c}\text { Tourniquet time } \\
\quad(\text { minutes) }\end{array}$ & $55(0-105)$ & $52(0-106)$ & $54(0-90)$ \\
\hline
\end{tabular}

$\mathrm{ASA}=$ American Society of Anesthesiologists.

homoscedastic (Levene's test, $\mathrm{p}>0.05$ for sensory and motor block durations) and normal $(\mathrm{p}>0.05)$, we used one-way ANOVA to compare duration across groups and the least significant difference test to compare duration between individual groups. Pain intensity was compared using the Kruskal-Wallis test among groups, followed by the corrected Mann-Whitney test between groups. Data are expressed as mean (SD) or median (minimum-maximum) unless indicated otherwise. Statistical analysis of data was performed with the SPSS software program (Version 11.0; SPSS Inc, Chicago, IL, USA).

\section{Results}

The addition of $20 \mathrm{mg}$ parecoxib locally on the nerve block prolonged the motor $(\mathrm{p}<0.001)$ and sensory block times $(\mathrm{p}=0.001)$ compared with the control group, whereas $20 \mathrm{mg}$ parecoxib intravenously affected neither the motor $(\mathrm{p}=0.3$ versus Group A) nor sensory $(\mathrm{p}=0.8$ versus Group A) block times (Table 3). Primary block effectiveness was comparable in the three groups (Table 3 ).

The addition of $20 \mathrm{mg}$ parecoxib locally on the nerve block and intravenously relieved $(\mathrm{p}<0.001)$ the pain intensity compared with the control group $[\mathrm{VAS}=83$ (median); 30-100 (minimum-maximum)], at 24 hours postoperatively. Incision pain VAS score in Group B was lower $(\mathrm{p}=0.042)$ [VAS $=40$ (median); 12-94 (minimum-maximum)] than for Group C [VAS $=63$ (median); 14-100 (minimum-maximum)].

During the 24-hour postoperative assessment, seven patients, three in Group A, two in Group B, and two in Group C, reported dysesthesia (Grade I according to the classification of Dindo et al. [8]). None of the patients required treatment, and the dysesthesia disappeared within
Table 3. Characteristics of block (mean $[\mathrm{SD}]$ )

\begin{tabular}{llll}
\hline Block & Group A & Group B & Group C \\
\hline Motor block time (minutes) & $371(139)$ & $509(131)^{*}$ & $414(132)$ \\
Sensory block time (minutes) & $439(140)$ & $543(132)^{*}$ & $457(132)$ \\
Primary block effectiveness & $96 \%$ & $96 \%$ & $96 \%$ \\
\hline
\end{tabular}

$* \mathrm{p}<0.05$ versus Group A; motor block time $=$ time between the end of block and complete recovery of motor function; sensory block time $=$ time between the end of block and complete recovery of sensation.

2 weeks in five of the patients. The other two patients reported that the dysesthesia had disappeared within 2 months after surgery. All seven patients had experienced paresthesia while the block was performed. The operating surgeon did not think the dysesthesia was attributable to the surgical procedure. Therefore we postulated that the dysesthesia was related to anesthesia complications. Arterial puncture during the axillary block had occurred in three of these patients (Grade I, one in each group). Other symptoms of anesthetic toxicity were not reported.

\section{Discussion}

Intravenous COX-2 antagonist may inhibit the systemic inflammatory response and provide postoperative pain relief [26]. Several studies [16, 17] have shown COX-2 in the spinal dorsal horn could modulate spinal nociceptive processes. Furthermore, epidural parecoxib reportedly has no neurotoxicity in rats over 21 days [15]. These findings suggested applying a COX-2 antagonist directly to the central or peripheral nerve might have better analgesic effect than if used intravenously. The purpose of this randomized controlled trial study was twofold: (1) using a multiple nerve stimulation technique, we compared the sensory and motor block times of an axillary brachial plexus nerve block of $0.25 \%$ ropivacaine with or without additional parecoxib; and (2) we evaluated the analgesic adjuvant effect of parecoxib to determine which regimen, injected locally on the brachial plexus nerve or injected intravenously, might be better.

Although a randomized trial, readers should be aware of the limitations of our study. First, there are some limitations of our enrollment criteria. Nociception and pain signal transduction are complicated processes in which there are many factors that may affect pain sensation. When enrolling patients, we excluded the definite painrelated main factors (eg, psychiatric anxiety and alcohol abuse) but did not limit other factors that also might affect pain sensation (eg, age and sex). If we had stricter enrollment criteria such as limiting the study to one certain type of surgical procedure or just one sex, the data would be 
more persuasive. Second, missing preoperative pain tolerance of enrolled patients is another defect of our study because we do not have any apparatus to determine the heat or pressure pain threshold for humans. Third, we used a single dose and did not examine the dose-effect relationship between parecoxib and the duration of the nerve block or pain relief. Nonetheless, we did find the addition of $20 \mathrm{mg}$ parecoxib to ropivacaine on the nerve block prolonged the blockade time and ameliorated postoperative pain intensity.

We found the addition of $20 \mathrm{mg}$ parecoxib to ropivacaine $(0.25 \%)$ locally on the axillary brachial plexus nerve block using a multiple-nerve stimulation technique prolonged the duration of the axillary brachial plexus blockade. The use of parecoxib as an adjuvant to local anesthetics on peripheral nerve blocks has not been described before, and its mechanism of action and its metabolism are not clearly understood. Several explanations may be possible for the prolonged blockade effect of parecoxib. First, vasoconstriction, an adjunctive effect of epinephrine on local anesthetics [19], is presumed responsible for peripheral nerve block prolongation. Selective COX-2 inhibition without COX-1 inhibition will preserve synthesis of the vasoconstrictive thromboxane $A_{2}$ and inhibit production of the vasodilator prostacyclin, therefore tipping the balance toward vasoconstriction and thrombosis [7]. Second, modification of the action of the local anesthetic in the sodium channel either directly or indirectly cannot be excluded as a mechanism [5]. Third, the axillary brachial plexus nerve block was performed using a multiple-nerve stimulation technique in the current study. Independent of the method, the proximity of the needle to the brachial nerves is important for a successful axillary nerve block. In the current experiment, the stimulating current was between 0.2 and $0.5 \mathrm{~mA}$, which meant that the stimulating needle was sufficiently close to the nerve. In a previous study, Zhao et al. reported needle position was considered adequate at the point when a motor response could be elicited and there was little risk of nerve injury by this stimulating current [30]. Under this circumstance, although the concentration of ropivacaine administrated in our study was $0.25 \%$, which was lower than the usual dosage of $0.5 \%$ or $0.75 \%$, the success rate was still higher than $90 \%$. This also may explain why an amount as low as $1 \mathrm{~mL}$ parecoxib in each nerve is sufficient for an adjunctive effect on the nerve blockade.

COX inhibitors reduce inflammation and hyperalgesia by decreasing prostaglandin (PG) production. In addition to systemic effects, the role of COX-2 involved in the central nervous system response is more important. Inflammation causes the induction of COX-2, leading to the release of prostanoids which sensitize peripheral nociceptor terminals and produce localized pain hypersensitivity [29].
Therefore, administration of a COX-2 antagonist on spinal or peripheral nerves may offer better pain relief than intravenous systemic absorption. Pinardi et al. [22] reported that intrathecal coadministration of $\mathrm{COX}-2$ inhibitors in mice substantially enhanced morphineinduced antinociception against visceral pain and could result in an opioid-sparing action. In clinical use, patients with postlaminectomy syndrome showed satisfactory pain relief with epidural indomethacin without neurologic deficits [2]. In our study, the adjuvant effect of $20 \mathrm{mg}$ parecoxib on the nerve block provided better incision pain management during the first 24 hours postoperatively than the same dose of parecoxib injected intravenously. The possible explanation might be that the adjuvant of parecoxib affected the absorption and action of the local anesthetics, or parecoxib had a direct action on a peripheral nerve and ganglion. Using a COX-2 inhibitor locally may release the inflammation induced by the injection, which could not be excluded. Further studies are needed to explore the precise mechanisms.

Establishing absence of neurotoxicity of parecoxib on a spinal or peripheral nerve block is important. Direct evidence supporting the use of epidural parecoxib was reported by Kim et al. [15] exploring possible neurotoxicity of epidural parecoxib in rats over 21 days. They observed no histologic neurotoxicity on the spinal cord or abnormalities in sensorimotor function or behavior in rats that received epidural parecoxib. We followed all patients for 2 months after surgery. No paresthesias were reported at that time. We believe the study of Kim et al. in addition to ours provides evidence for the safety and feasibility of using parecoxib on the spinal cord or peripheral nerve, although further studies using larger animal species are necessary to assess the full therapeutic scope and detailed safety of parecoxib.

There is a considerable difference in practicing and treating these orthopedics patients in different countries. For example, in the United States, most of the patients are given a prescription for pain medication or use over-thecounter Tylenol ${ }^{\mathbb{R}}$ (McNeil-PPC Inc, Fort Washington, PA, USA) preoperatively. However in our country, analgesic medication before surgery is not routinely prescribed. Most patients having a total hip replacement will not stay in the hospital longer than 5 days in the United States, but in Pakistan, the median length of a hospital stay for patients undergoing the same surgery is longer than 10 days [1]. These differences in treating patients may be attributed to disparate medical service standards, economic situations, and hospital scales in different countries.

We found the addition of $20 \mathrm{mg}$ parecoxib to ropivacaine on an axillary brachial plexus block resulted in prolongation of the blockade and improved postoperative pain management with no evident side effects. In addition 
to our study, there are a couple studies verifying the efficiency or safety of administration of COX-2 antagonist to the spinal cord or peripheral nerves. This new regimen may be more preferable than parecoxib injected intravenously for forearm orthopaedic surgery. More research will be performed to reveal the dose-effect relationship, mechanism of action, metabolism, and long-term safety.

Acknowledgments We acknowledge the assistance of Ximing Chen PhD for the study design and statistical analysis and Qin Ying AS for drug and solutions preparations. We also acknowledge the assistance of Tyler Andrew Long BA for help editing the manuscript.

\section{References}

1. Abbas K, Umer M, Qadir I, Zaheer J, ur Rashid H. Predictors of length of hospital stay after total hip replacement. J Orthop Surg (Hong Kong). 2011;19:284-287.

2. Aldrete JA. Epidural injections of indomethacin for postlaminectomy syndrome: a preliminary report. Anesth Analg. 2003;96:463-468.

3. Andersson A, Akeson J, Dahlin LB. Efficacy and safety of axillary brachial plexus block for operations on the hand. Scand $J$ Plast Reconstr Surg Hand Surg. 2006;40:225-229.

4. Brandsma JW, Schreuders TA, Birke JA, Piefer A, Oostendorp R. Manual muscle strength testing: intraobserver and interobserver reliabilities for the intrinsic muscles of the hand. $J$ Hand Ther. 1995;8:185-190.

5. Butterworth JF 4th, Strichartz GR. Molecular mechanisms of local anesthesia: a review. Anesthesiology. 1990;72:711-734.

6. Daniels SE, Grossman EH, Kuss ME, Talwalker S, Hubbard RC. A double-blind, randomized comparison of intramuscularly and intravenously administered parecoxib sodium versus ketorolac and placebo in a post-oral surgery pain model. Clin Ther. 2001; 23:1018-1031.

7. Day RO, Graham GG. The vascular effects of COX-2 selective inhibitors. Aust Prescr. 2004;27:142-145.

8. Dindo D, Demartines N, Clavien PA. Classification of surgical complications: a new proposal with evaluation in a cohort of 6336 patients and results of a survey. Ann Surg. 2004; 240: 205-213.

9. Duma A, Urbanek B, Sitzwohl C, Kreiger A, Zimpfer M, Kapral S. Clonidine as an adjuvant to local anaesthetic axillary brachial plexus block: a randomized, controlled study. $\mathrm{Br} J$ Anaesth. 2005;94:112-116.

10. Giorgi M, Saccomanni G, Del Carlo S, Manera C, Lavy E. Pharmacokinetics of intravenous and intramuscular parecoxib in healthy Beagles. Vet J. 2012;193:246-250.

11. Gonano C, Kettner SC, Ernstbrunner M, Schebesta K, Chiari A, Marhofer P. Comparison of economical aspects of interscalene brachial plexus blockade and general anaesthesia for arthroscopic shoulder surgery. Br J Anaesth. 2009;103:428-433.

12. Graff J, Arabmotlagh M, Cheung R, Geisslinger G, Harder S. Effects of parecoxib and dipyrone on platelet aggregation in patients undergoing meniscectomy: a double-blind, randomized, parallel-group study. Clin Ther. 2007;29:438-447.

13. Kapral S, Gollmann G, Waltl B, Likar R, Sladen RN, Weinstabl $\mathrm{C}$, Lehofer F. Tramadol added to mepivacaine prolongs the duration of an axillary brachial plexus blockade. Anesth Analg. 1999;88:853-856.

14. Keats AS. The ASA classification of physical status: a recapitulation. Anesthesiology. 1978;49:233-236.

15. Kim YH, Lee PB, Park J, Lim YJ, Kim YC, Lee SC, Ahn W. The neurological safety of epidural parecoxib in rats. Neurotoxicology. 2011;32:864-870.

16. Koppert W, Wehrfritz A, Körber N, Sittl R, Albrecht S, Schüttler $\mathrm{J}$, Schmelz M. The cyclooxygenase isozyme inhibitors parecoxib and paracetamol reduce central hyperalgesia in humans. Pain. 2004;108:148-153.

17. Martin F, Fletcher D, Chauvin M, Bouhassira D. Constitutive cyclooxygenase- 2 is involved in central nociceptive processes in humans. Anesthesiology. 2007;106:1013-1018.

18. Maser RE, Nielsen VK, Bass EB, Manjoo Q, Dorman JS, Kelsey SF, Becker DJ, Orchard TJ. Measuring diabetic neuropathy: assessment and comparison of clinical examination and quantitive sensory testing. Diabetes Care. 1989;12:270-275.

19. Newton DJ, McLeod GA, Khan F, Belch JJ. The effect of adjuvant epinephrine concentration on the vasoactivity of the local anesthetics bupivacaine and levobupivacaine in human skin. Reg Anesth Pain Med. 2004;29:307-311.

20. Niruthisard S, Werawataganon T, Bunburaphong $P$, Ussawanophakiat M, Wongsakornchaikul C, Toleb K. Improving the analgesic efficacy of intrathecal morphine with parecoxib after total abdominal hysterectomy. Anesth Analg. 2007;105:822-824.

21. Noveck RJ, Laurent A, Kuss M, Talwalker S, Hubbard RC. Parecoxib sodium does not impair platelet function in healthy elderly and non-elderly individuals: two randomised, controlled trials. Clin Drug Investig. 2001;21:465-476.

22. Pinardi G, Prieto JC, Miranda HF. Analgesic synergism between intrathecal morphine and cyclooxygenase-2 inhibitors in mice. Pharmacol Biochem Behav. 2005;82:120-124.

23. Rasmussen GL, Steckner K, Hogue C, Torri S, Hubbard RC. Intravenous parecoxib sodium for acute pain after orthopedic knee surgery. Am J Orthop (Belle Mead NJ). 2002;31:336-343.

24. Rodriguez J, Taboada M, Del Rio S, Barcena M, Alvarez J. A comparison of four stimulation patterns in axillary block. Reg Anesth Pain Med. 2005;30:324-328.

25. Sia S, Bartoli M, Lepri A, Marchini O, Ponsecchi P. Multipleinjection axillary brachial plexus block: a comparison of two methods of nerve localization-nerve stimulation versus paresthesia. Anesth Analg. 2000;91:647-651.

26. Simon LS. Role and regulation of cyclooxygenase-2 during inflammation. Am J Med. 1999;106:37S-42S.

27. Stan TC, Krantz MA, Solomon DL, Poulos JG, Chaouki K. The incidence of neurovascular complications following axillary brachial plexus block using a transarterial approach: a prospective study of 1,000 consecutive patients. Reg Anesth. 1995;20: 486-492.

28. Stoltz RR, Harris SI, Kuss ME, LeComte D, Talwalker S, Dhadda S, Hubbard RC. Upper GI mucosal effects of parecoxib sodium in healthy elderly subjects. Am J Gastroenterol. 2002;97:65-71.

29. Svensson CI, Yaksh TL. The spinal phospholipase-cyclooxygenase-prostanoid cascade in nociceptive processing. Annu Rev Pharmacol Toxicol. 2002;42:553-583.

30. Zhao X, Wang YW, Chen H, Zhang Z, You XM, Luo K, Jin YJ. Efficacy of low dose levobupivacaine $(0.1 \%)$ for axillary plexus block using multiple nerve stimulation. Anaesth Intensive Care. 2008;36:850-854. 